\title{
Rare artifact in IOPA: A miniature UFO
}

\author{
Anusha R. L. ${ }^{1, *}$, Nilofer Halim², Chaithra Kalkur ${ }^{3}$, Sham Kishor Kannepady ${ }^{4}$ \\ ${ }^{1,3}$ Reader, ${ }^{2}$ Assistant Professor, ${ }^{4}$ Senior Lecturer, ${ }^{1-3}$ Dept. of Oral Medicine \& Radiology, Century Dental College, Poinachi, \\ Kerala, ${ }^{4}$ Division of Oral Diagnostics \& Surgical Sciences, International Medical University, Lampur, Malaysia
}

*Corresponding Author:

Email: anisharl@gmail.com

\begin{abstract}
Dental radiographs play very crucial role in comprehensive patient care. In dentistry, a radiographic examination is essential for diagnostic purposes. An ideal radiograph is one that provides a great deal of information, the image exhibits proper density and contrast, has sharp outlines and is of the same shape and size as the object being radiographed. But most of the time, we might not get to see ideal radiographs. Poor radiographs contribute to a loss of diagnostic information. It might be because of any radiographic faults or artifacts. An artifact is a structure or an appearance that is not normally present on the radiograph and is produced by artificial means. We are hereby reporting an incidental rare type of artifact resembling as a miniature Unidentified Flying Object (UFO) during routine dental radiography.
\end{abstract}

Keywords: Artifact, Metal, IOPA, UFO.

\section{Introduction}

A dental radiograph is a photographic image produced on film by the passage of $\mathrm{x}$-rays through teeth and supporting structures. The dental radiographer must have a thorough understanding of the value and importance of dental radiographs. In addition, the dental radiographer must be familiar with the uses of dental radiographs, the benefits of dental radiographs, and the information that can be found on dental radiographs. ${ }^{1}$

Radiographic interpretation is an essential part of diagnostic process. The ability to recognise what is revealed by a radiograph enables a dental professional to play a vital role in the detection of various diseases, lesions and conditions of the jaws that cannot be identified clinically. ${ }^{2}$ A profound knowledge of the variation of normal appearance is required to be able to recognize an abnormal appearance. ${ }^{3}$ In order to correctly and competently interpret a radiograph, the dental professional must be well versed in identification and recognition of the normal anatomical structures and their variations seen in the radiographs taken of the various regions. $^{2}$

HM Worth defined an ideal radiograph as "It is one which has desired density and overall blackness and which shows the part completely without distortion with maximum details and has the right amount of contrast to make the details fully apparent". ${ }^{3}$ According to Principles of radiographic interpretation, the radiographer should not interpret any faulty radiographs. ${ }^{3}$ This article highlights about a radiopaque object observed during routine dental radiography, which appeared like a miniature unidentified flying object (UFO) and we have also discussed the various causes of artifacts and emphasis on the identification of such artifacts, thereby misdiagnosing it as a pathology.

\section{Case Report}

A 30 year old female patient reported to the Department of Oral Medicine and Radiology complaining of pain in the lower right back tooth since 3 days. Pain was of sudden onset, pricking type, severe, radiating to the right forehead region. Her medical history was non-contributory. Past dental history revealed history of restoration. She was of well-built and nourished with all vital signs within normal limits. Submandibular lymphadenopathy was noticed. On intra-oral examination, multiple root stumps were seen with restored teeth of right and left mandibular second molar. Provisional diagnosis of acute irreversible pulpitis of right mandibular second molar was considered and was advised for routine Intra Oral Periapical Radiograph (IOPAR). The IOPAR (Fig. 1) suggested two root pieces with ill-defined periapical radiolucency suggestive of periapical abscess in right mandibular second molar. But mean time, we noticed a well-defined radiopaque structure superimposing on the roots of third molar, which was circular with small three spike like projections from its base resembling like a miniature UFO. Radiographic differential diagnosis of a metallic artifact was considered followed by amalgam tattoo, other restorative material, metallic brackets. To rule out the possibility of metallic artifact, we repeated the radiograph. The second radiograph did not show any radiopaque structure (Fig. 2). Later, we visualized the patient carefully to see any metallic object present in the head and neck region which might have accidentally exposed during the radiography. We found that the patient was a muslim female and had placed a small clip on the cheek region to tie a scarf on her head. That metallic clip was the artifact seen on the first radiograph mimicking as a miniature UFO. We prescribed antibiotics and analgesics for 5 days and recalled the patient for extraction of root stumps. 


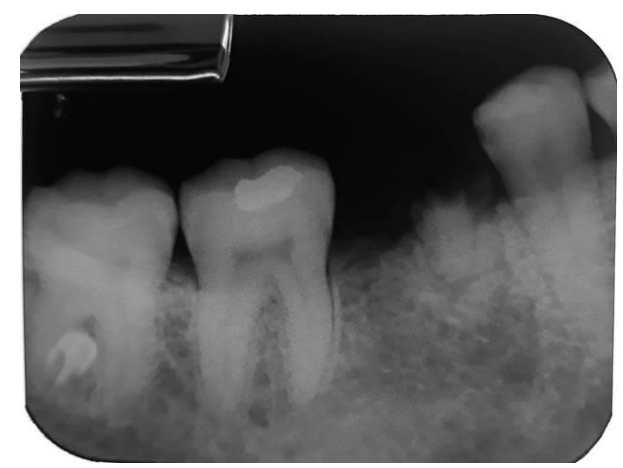

Fig. 1: The IOPAR shows two root pieces with illdefined periapical radiolucency suggestive of periapical abscess in right mandibular second molar. A well-defined radiopaque structure superimposing on the roots of third molar, which was circular with small three spikes like projections from its base resembling like a miniature UFO was also noticed

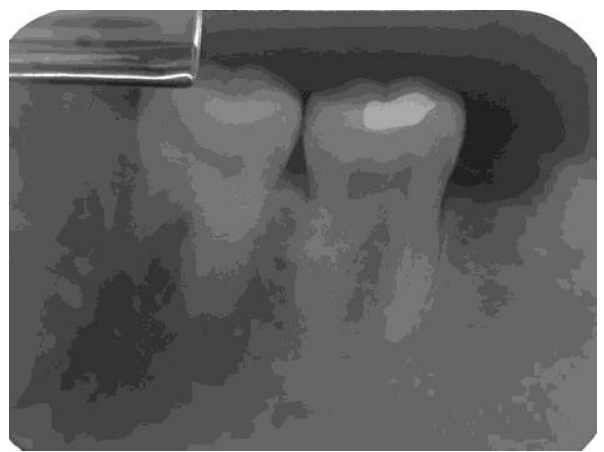

Fig. 2: The second IOPAR showing absence of the metallic object

\section{Discussion}

The International Dictionary of Medicine and Biology7 define an artifact as "any record or image obtained in the course of applying a medical diagnostic technique which is not representative of the structures under study but is adventitious".

Artifacts on radiographs are permanent, unwanted, extraneous marks which detract from the general quality of radiographs. They may mimic or obscure abnormalities and cause errors in radiographic diagnosis.

Radiographic errors may be due to errors related to positioning errors or related to all aspects of processing (processing errors), exposure errors (altered Kvp or $\mathrm{mA}$ ), improper exposure time, improper handling of film packet. It might be because of the movement of the x-ray tube head or patient's movement or film displacement. $^{4}$

The dental appliances, tongue piercings or any body piercing in head and neck region, metallic ear rings or nose rings or neck chains or any metallic jewellery, metallic hairpins if not removed during the routine radiography, it will be recorded and appear superimposed over the dental images on the final radiograph, which appears radiopaque. ${ }^{5}$ So the patient is advised to remove dental appliances, such as partial and complete dentures before taking any radiographs. Whereas for extraoral radiography (such as panoramic radiographs) all metallic objects has to be removed from head and neck region such as wigs, hairpieces, eyebrow jewellery, necklaces and napkin chains must be removed. ${ }^{5}$ In the present case, we had advised intraoral periapical radiograph of mandibular molar. Usually no metallic objects get exposed in that region. But we noticed a well-defined radiopaque artifact in the final radiograph, which made us to re-examine the patient properly to rule out any artifact in that region. To our surprise, it was patient's metallic small pin or clip which she had put on the head scarf. So this mistake enlightened and enforces us to follow proper and strict examination of the patient before routine dental radiography.

\section{Conclusion}

Dental radiology is a valuable adjunct in diagnosis and treatment planning. Any radiographic faults or artifacts make the radiograph undiagnostic. The dentists should also be aware of the most common artifacts seen in dental radiography and should have thorough knowledge about the normal anatomical landmarks so that he can differentiate from the artifacts. This article enforces all the dental technicians and radiographers for a strict and proper examination of the patient before routine dental radiography to avoid superimposition of any artifacts thereby not hindering the radiograph to be undiagnostic.

\section{References}

1. Haring and Howerton. Dental Radiography: Principles and Techniques, third edition. Elsevier 2006:141.

2. Freny R Karjodkar. Essentials of oral and maxillofacial radiology. First edition. Jaypee Brothers 2014:47.

3. White, Staurt C., and M. J Pharoah. Oral Radiology: Principles and interpretation. St. Louis, Mo: Mosby/Elsevier 2009.

4. Dr. Cheena Singh1 and Dr. Kamal sagar. Artifacts in Dental Radiography: A Mini Review. Int J Adv Res 2016;4(9):95860.

5. Olaf E. Langland, Robert P. Langlias and John W. Preece. Principles of Dental Imaging. Second edition. Lippincott Williams and Wilkins 2002. 